\title{
Influence of Linear Alkyl Benzene Sulphonate on Corrosion of Iron in Presence of Magnetic Field: Kinetic and Thermodynamic Parameters
}

\author{
A. K. Maayta, ${ }^{1}$ Mohammad M. Fares, ${ }^{1}$ and Ali F. Al-Shawabkeh ${ }^{2}$ \\ ${ }^{1}$ Department of Applied Chemistry, Jordan University of Science and Technology, P.O. Box 3030, Irbid 22110, Jordan \\ ${ }^{2}$ Department of Applied Sciences, Faculty of Engineering Technology, Al-Balqa Applied University, P.O. Box 15008, \\ Amman 11134, Jordan
}

Correspondence should be addressed to Mohammad M. Fares, fares@just.edu.jo

Received 24 February 2010; Revised 11 August 2010; Accepted 11 September 2010

Academic Editor: Jerzy A. Szpunar

Copyright ( $) 2010$ A. K. Maayta et al. This is an open access article distributed under the Creative Commons Attribution License, which permits unrestricted use, distribution, and reproduction in any medium, provided the original work is properly cited.

\begin{abstract}
Implementation of linear alkyl benzene sulphonate, LAS, as corrosion inhibitor on the surface of iron metal in acidic media and in the absence and presence of magnetic field has been investigated. Adsorption of inhibitor molecules on iron surface showed Langmuir adsorption isotherms fit in acidic media. In the absence of magnetic field, apparent activation energy values $\left(E_{a}\right)$ in $2.0 \mathrm{M} \mathrm{HCl}$ solutions provide evidence of the inhibitory effect of LAS on iron; similarly, the enthalpy of activation values $\left(\Delta H^{\neq}\right)$ was in accordance with activation energy values. Apparent entropy of activation shows that at larger inhibitor concentration, solvent entropy decrease in $1.0 \mathrm{M} \mathrm{HCl}$ and solvent entropy increase in $2.0 \mathrm{M} \mathrm{HCl}$ were obtained. Furthermore, spontaneity, through equilibrium constant $\left(K_{\text {ads. }}\right)$ values and free energy value $\left(\Delta G_{\text {ads. }}\right)$ of the adsorption process, shows a drastic decrease upon temperature increase. In the presence of magnetic field, lower rates of corrosion, larger activation energies, solvent entropy increase, larger equilibrium constant $\left(K_{\text {ads. }}\right)$ value, and lower free energy value $\left(\Delta G_{\text {ads. }}\right)$ have been investigated.
\end{abstract}

\section{Introduction}

Corrosion Inhibitors are one of the possible means to suppress corrosion through metal surface isolation from corrosive agents. Among the best corrosion inhibitors that act as major adsorption centers are some organic compounds, containing functional electronegative groups and $\pi$-electron in triple or conjugated double bonds. These hetero-atoms, such as sulfur, phosphorus, nitrogen, and oxygen, together with heterocyclic or conjugated aromatic, play significantly in inhibition process [1-4]. Such polar and nonpolar groups regarded as the reaction centre for adsorption processes exhibit strong affinity for metal surfaces and thus strongly act as corrosion inhibitors $[5,6]$. The efficiency of these inhibitors mainly depends on their ability to be adsorbed on the metal surface that results with replacement of water molecules and are highly dependent on presence of $\pi$ conjugated and hetero-atomic structures [7-10]. Generally, the adsorption layer is considered as shielding layer that prevents metal from corrosion.

Implementation of magnetic field on metal surfaces in aqueous solutions can promote corrosion resistance and provide some new properties and better characteristics including microroughness, hydrophilicity, and microtopography modification through lowering microroughness and minimizing the actual surface area in micro and nanoscales [11]. The effects of an applied magnetic field were extended to affect electrochemical reactions through mass transfer enhancement, electro-deposition quality improvement, potential distribution and current control, and reduction of corrosion rates [12-17].

The aim of this work is to study and implement linear alkyl benzene sulphonate surfactant, LAS, as a corrosion inhibitor on the surface of iron metal in the absence and presence of magnetic field in acidic media. Kinetic and thermodynamic parameters were investigated to give deeper 


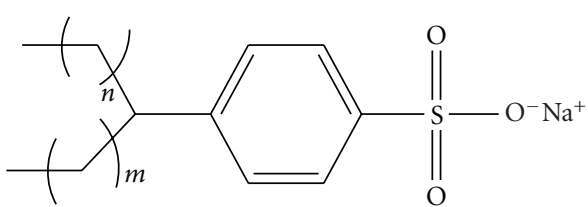

Scheme 1: Chemical structure of LAS inhibitor, $n+m=7-10$ methyl units.

insight to the effect of inhibitor structure and magnetic field of the inhibition process. Two techniques were used to study the inhibition process in presence and absence of magnetic field in 1.0 to $4.0 \mathrm{M} \mathrm{HCl}$ concentration range, namely, weight loss measurements and hydrogen evolution method. The corrosion study was performed in acidic medium due to its dramatic effect on the enhancement of iron corrosion.

LAS surfactant contains long $\mathrm{CH}_{2}$ groups as hydrophobic tail bounded to benzene and sulphonate group as conjugated-polar head, which presents typical inhibitor example that contains $\pi$-conjugated and hetero-atomic structures (Scheme 1). The critical micelle concentration of $\mathrm{C}_{13}$ LAS was determined as $112 \mathrm{ppm}$ [18].

\section{Experimental}

2.1. Materials. The iron $(\mathrm{Fe})$ was used in the form of sheets with $30 \times 10 \times 0.5 \mathrm{~mm}$ dimensions which were degreased with acetone, polished, dried, and weighed until used. LAS inhibitor (molar mass $=346 \mathrm{~g} / \mathrm{mol}$ ), $88 \%$ by mass, supplied by Sulpho-chemical Company, Jordan, was diluted to the appropriate concentrations using triple distilled water. Magnetic field was supplied by two magnet plates with $7.0 \times$ $7.0 \times 1.9 \mathrm{~cm}$ dimensions. The strength of magnetic field was measured to be $100 \mathrm{mT}$ using Holl-Probe, Leybold-Hereaus instrument.

2.2. Weight Loss Measurements. The Fe-sheets were immersed in $10 \mathrm{ml}$ of $1.0 \mathrm{M} \mathrm{HCl}$ that contains different concentrations once at a time of LAS inhibitor $(200,400$, 600,800 , and $1000 \mathrm{ppm}$ ) solutions for 1.0 hour at $25^{\circ} \mathrm{C}$. After reaction time was complete, the iron sheets were washed and rinsed thoroughly in distilled water, cleaned with a soft cloth, and dried to constant weight. The process was repeated using $2.0 \mathrm{M}, 3.0 \mathrm{M}$, and $4.0 \mathrm{M} \mathrm{HCl}$ solutions at the same temperature. For magnetic field measurements, the test tube containing the $\mathrm{Fe}$-sheet in the specified $\mathrm{HCl}$ solution was placed between two magnets that supply $100 \mathrm{mT}$ magnetic strength.

Likewise, in kinetic measurements the Fe-sheets were immersed in $1.0 \mathrm{M}$ and $2.0 \mathrm{M} \mathrm{HCl}$ using the same inhibitor concentrations at elevated temperatures (i.e., 35 and $45^{\circ} \mathrm{C}$ ) using different time intervals (i.e., $1.0 \mathrm{~h}, 2.0 \mathrm{~h}, 3.0 \mathrm{~h}$, and $4.0 \mathrm{~h}$ ), where all measurements were done in absence of magnetic field. The inhibition efficiency (\%IE) was calculated as follows:

$$
\% \mathrm{IE}=\frac{W_{0}-W_{i}}{W_{0}} \times 100
$$

where $w_{0}$ and $w_{i}$ are corrosion weight losses of iron in uninhibited and inhibited solutions, respectively. The rate of corrosion $\left(R_{C}\right)$ of iron was calculated (in $\mathrm{mg} / \mathrm{cm}^{2} . \mathrm{sec}$ ) using the following relation:

$$
R_{c}=\frac{W}{S t},
$$

where $W$ is weight loss, $S$ is area of Fe-sheet, and $t$ is the exposure time of specimens. All weight measurements were performed using semimicron balance (Saratorius $2024 \mathrm{MPb}$ ) with doublicated replications and a precision of $\pm 0.01 \mathrm{mg}$.

2.3. Hydrogen Evolution Method. Fe-sheets were incubated in specific concentration of LAS and $\mathrm{HCl}$ solution in $10 \mathrm{ml}$ test tube, sealed and connected to a thin $P V C$ tube to enable hydrogen gas collection over water by graduated cylinder according to

$$
\mathrm{Fe}(\mathrm{s})+3 \mathrm{HCl}(\mathrm{aq}) \longrightarrow \mathrm{FeCl}_{3}(\mathrm{aq})+\frac{3}{2} \mathrm{H}_{2}(\mathrm{~g}) .
$$

2.4. Adsorption Isotherms. $\theta$ is the degree of surface coverage of inhibitor on iron sheet surface, which was calculated using the following relation:

$$
\theta=\frac{W_{0}-W_{i}}{W_{0}}
$$

Different adsorption isotherms would be used such as, Tempkin [19, 20], Langmuir [21], and Frumkin [22] to elucidate data and consequently choose the most fitting isotherm that has the best regression coefficients, $R^{2}$ (i.e., very close to 1.0 ).

\section{Results and Discussions}

\subsection{Weight Loss Measurements}

3.1.1. In Absence of Magnetic Field. Figures 1(a) and 1(b) show weight loss and evolved volume of hydrogen gas versus concentration of LAS inhibitor, in ppm, respectively. It could be seen that as concentration of LAS inhibitor increases, linear decrease in weight loss and volume of $\mathrm{H}_{2}$ gas were observed. The concentrations of LAS used were above its critical micelle concentration (i.e., $112 \mathrm{ppm}$ ) to make sure that inhibitor is in the form of either spheres or rods and thus can preferably be adsorbed on the surface of the metal. Furthermore, the increase in $\mathrm{HCl}$ concentration used was found to affect the weight loss and $\mathrm{H}_{2}$ gas evolution processes. Figure 1(c) illustrates the linear increase of inhibition efficiency with LAS concentration at different molar $\mathrm{HCl}$ cocntrations. It could be seen that linear increase of inhibition efficiency was observed at increasing LAS concentration. The maximum inhibitory effects using $1000 \mathrm{ppm}$ LAS at $1.0 \mathrm{M}$ and $4.0 \mathrm{M} \mathrm{HCl}$ solution were equal to $\% \mathrm{IE}=23.1 \%$ and $\% \mathrm{IE}=15.4$, respectively. The capability of LAS inhibitor to prevent iron sheet from corrosion could be explained through the formation of electrostatic interactions between negatively charged sulphonate groups 


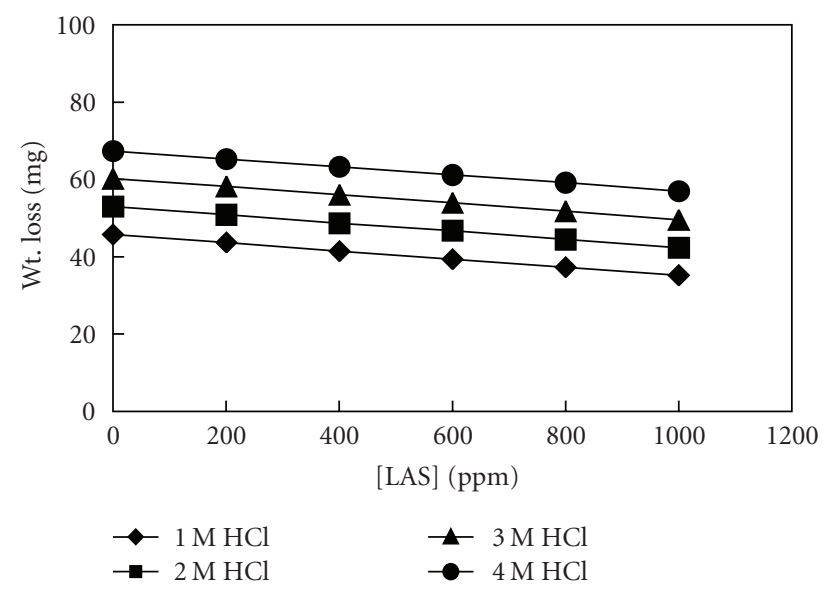

(a)

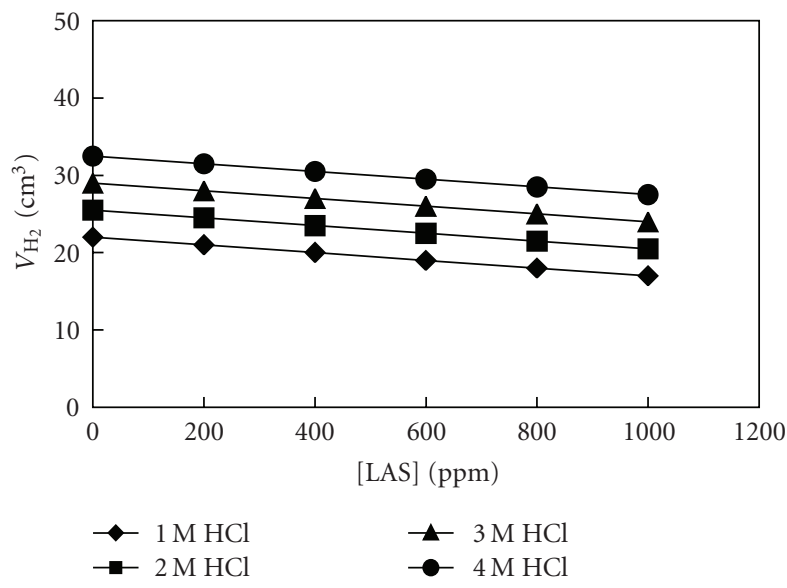

(b)

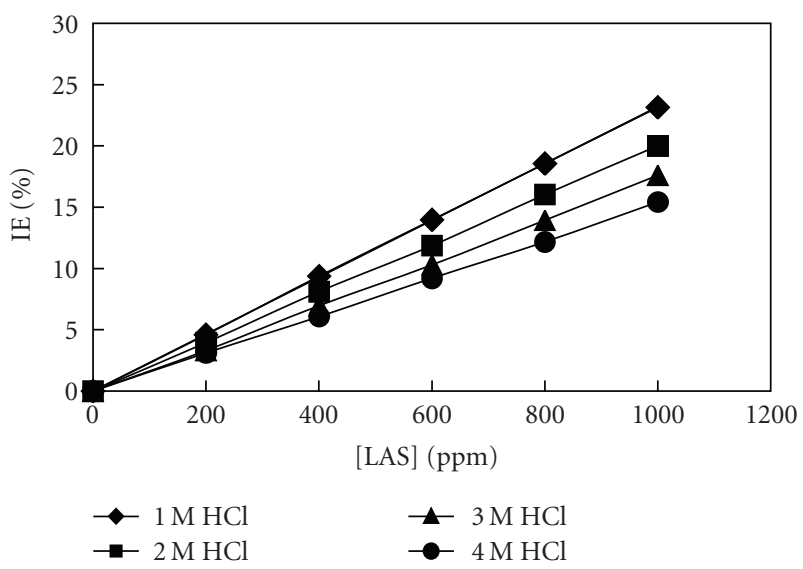

(c)

Figure 1: Change of (a) weight loss, (b) volume of $\mathrm{H}_{2}$ gas, and (c) Inhibition efficiency (\%IE) with LAS concentration using different $\mathrm{HCl}$ concentrations in absence of magnetic field for 1.0 hour exposure at $20^{\circ} \mathrm{C}$.

and positively Iron(II) and Iron(III) groups on the surface of the sheet. Additionally, Iron can act as Lewis acid, and hence might form coordinative bond interactions with lonepairs of oxygen atoms in sulphonate group which might act as Lewis base.

Furthermore, $\pi-\pi$ stacking interactions between benzene rings and hydrophobic interactions of long $\mathrm{CH}_{2}$ tail groups of different LAS molecules may take place. Ultimately, this would lead to formation of accumulated and well-arranged layers near and above each other on the surface of iron. Thus as concentration of LAS increases, expected more shielding and more inhibitory effect would result as Figures 1(a), 1(b), and $1(\mathrm{c})$ sugget.

3.1.2. In Presence of Magnetic Field. Figures 2(a) and 2(b) illustrate weight loss and evolved volume of hydrogen gas at different LAS inhibitor concentration in presence of magnetic field. Similar to Figure 1, the weight loss and hydrogen gas evolution were decreased with inhibitor concentration increase, and molar $\mathrm{HCl}$ concntation decrease. In the presence of magnetic field, it could be clearly seen that maximum inhibitory effects using $1000 \mathrm{ppm}$ LAS at $1.0 \mathrm{M}$ and $4.0 \mathrm{M} \mathrm{HCl}$ solution were $\% \mathrm{IE}=28.8 \%$ and $\% \mathrm{IE}=19.0$, respectively. Upon comparison of inhibition efficiencies (\%IE) in presence and absence of magnetic field, it appeared that there are always shifts of around $5 \%$ higher in the presence of magnetic field in nearly all measurements. This increase would be attributed entirely to the role of magnetic field in formation of well-oriented and well-ordered surfactant chains near and above each other on the metal surface. Magnetic field can induce a force called Lorentz force on a point charge. The point charge particle (i.e., negatively sulphonate groups) will be accelerated in the same linear orientation as the $\mathbf{E}$ field, but will curve perpendicularly to both the instantaneous velocity vector $\mathbf{v}$ and the $\mathbf{B}$ field according to the right-hand rule. Hence magnetic field could aid in surfactant chain alignment, creates better shielding, least entropy, and better inhibition processes.

3.2. Kinetic and Thermodynamic Corrosion Parameters. Arrhenius plot have been applied successfully on corrosion 


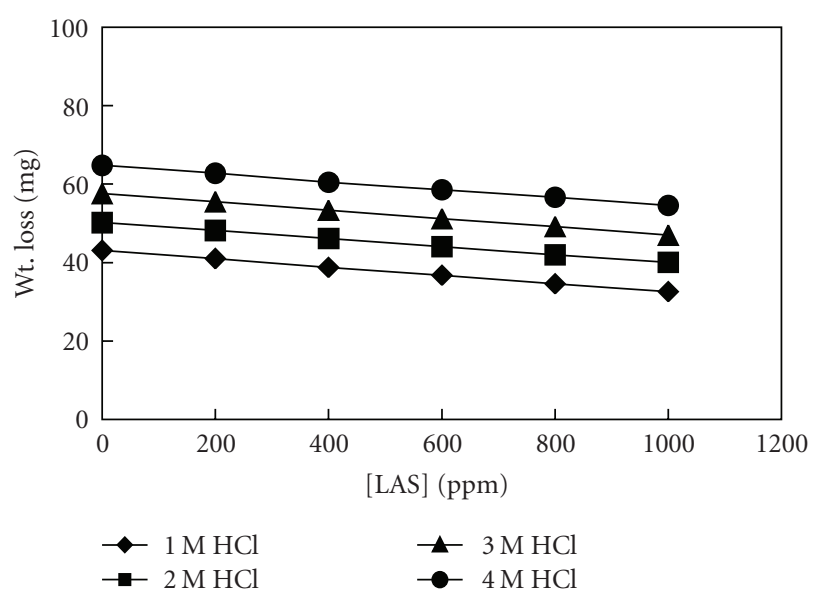

(a)

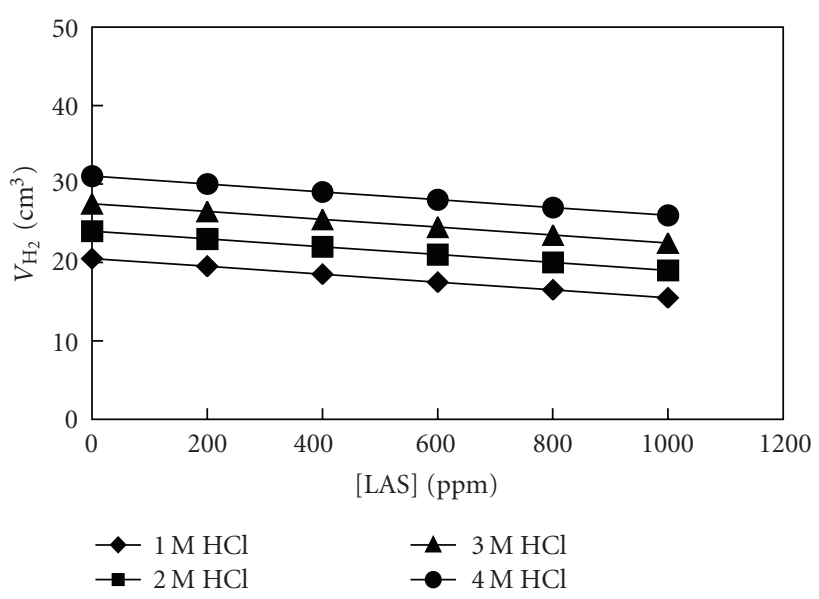

(b)

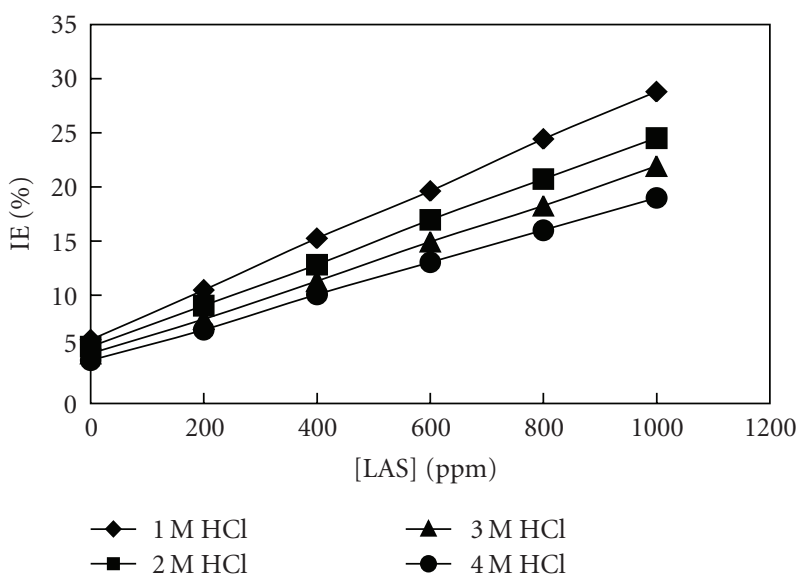

(c)

FIgure 2: Change of (a) weight loss, (b) Volume of $\mathrm{H}_{2}$ gas, and (c) Inhibition efficiency (\%IE) with LAS concentration using different $\mathrm{HCl}$ concentrations in presence of magnetic field for 1.0 hour exposure at $20^{\circ} \mathrm{C}$.

processes to determine kinetic parameters such as, preexponential factor and apparent activation energy of corrosion process using the following relationship [23-25]:

$$
\ln R_{C}=\ln A-\frac{E_{a, C}}{\mathrm{RT}},
$$

where $R_{C}$ is corrosion rate as determined using (2), $A$ is preexponential factor, $E_{a, C}$ is the activation energy for corrosion process, and $T$ is the absolute temperature. Furthermore, Eyring equation $[25,26]$ could be also used to determine some thermodynamic parameters using the following equation:

$$
\frac{R_{C}}{T}=\frac{k_{B}}{h} \exp \left(\frac{\Delta S^{\neq}}{R}\right) \exp \left(-\frac{\Delta H^{\neq}}{\mathrm{RT}}\right),
$$

where $k_{B}$ is Boltzmann constant, $h$ is the Planck constant, $\Delta H^{\neq}$is the enthalpy of activation, and $\Delta S^{\neq}$is the entropy of activation. As a result, a plot of $\ln \left(R_{C} / T\right)$ versus $1 / T$ would determine $\Delta H^{\neq}$and $\Delta S^{\neq}$from the slope and intercept, respectively.
3.2.1. In Absence of Magnetic Field. Table 1 summarizes the kinetic and thermodynamic parameters determined using Arrhenius and Eyring equations in the absence of magnetic field. In the absence of magnetic field, it could be clearly seen that the rate of corrosion increases with increasing temperature. The Arrhenius plot (Figure 3) could show the apparent activation energy values for increasing concentration of inhibitor. Such values were relatively in accordance with some literature results $[25,27,28]$, which confirm the chemisorptive nature of the interactions between the iron metal surface and the inhibitor structure. The activation energies at 200,600, and $1000 \mathrm{ppm}$ were higher than that at $0.0 \mathrm{ppm}$ (i.e., no inhibitor used) in $2.0 \mathrm{M} \mathrm{HCl}$, whereas it was lower in $1.0 \mathrm{M} \mathrm{HCl}$. This oscillatory behavior of activation energies confirm that concentration of inhibitor is not the only factor that affects the inhibition process. Actually, the effect of temperature has a dominant effect on inhibition process, the elevated temperature weakens, loosens and eventually destroys the inter- and intramolecular interactions between the surface of the metal and inhibitor structure, leaving only electrostatic interactions (i.e., Fe$\left.\mathrm{O}_{3} \mathrm{~S}-\right)$ bounded on the surface of the metal with free 
TABLE 1: Kinetic and thermodynamic parameters of corrosion process in $\mathrm{HCl}$ solution in the absence of magnetic field.

\begin{tabular}{|c|c|c|c|c|c|c|}
\hline$[\mathrm{HCl}](\mathrm{M})$ & [LAS] $(\mathrm{ppm})$ & $\mathrm{T}\left({ }^{\circ} \mathrm{C}\right)$ & $R_{C}\left(\mathrm{mg} / \mathrm{cm}^{2} \cdot \mathrm{sec}.\right)$ & $E_{a}(\mathrm{~kJ} / \mathrm{mol})$ & $\Delta H^{\neq}(\mathrm{kJ} / \mathrm{mol})$ & $\Delta S^{\neq}(\mathrm{J} / \mathrm{mol} \cdot \mathrm{K})$ \\
\hline \multirow{4}{*}{1.0} & \multirow{4}{*}{0.0} & 20 & $1.3 \times 10^{-4}$ & \multirow{4}{*}{92.3} & \multirow{4}{*}{89.6} & \multirow{4}{*}{-13.9} \\
\hline & & 30 & $5.2 \times 10^{-4}$ & & & \\
\hline & & 40 & $1.6 \times 10^{-3}$ & & & \\
\hline & & 50 & $4.5 \times 10^{-3}$ & & & \\
\hline \multirow{4}{*}{1.0} & \multirow{4}{*}{200} & 20 & $1.6 \times 10^{-4}$ & \multirow{4}{*}{86.1} & \multirow{4}{*}{83.1} & \multirow{4}{*}{-33.7} \\
\hline & & 30 & $5.1 \times 10^{-4}$ & & & \\
\hline & & 40 & $1.8 \times 10^{-3}$ & & & \\
\hline & & 50 & $4.0 \times 10^{-3}$ & & & \\
\hline \multirow{4}{*}{1.0} & \multirow{4}{*}{600} & 20 & $2.5 \times 10^{-4}$ & \multirow{4}{*}{77.9} & \multirow{4}{*}{74.9} & \multirow{4}{*}{-58.8} \\
\hline & & 30 & $5.9 \times 10^{-4}$ & & & \\
\hline & & 40 & $1.9 \times 10^{-3}$ & & & \\
\hline & & 50 & $4.7 \times 10^{-3}$ & & & \\
\hline \multirow{4}{*}{1.0} & \multirow{4}{*}{1000} & 20 & $1.7 \times 10^{-4}$ & \multirow{4}{*}{90.1} & \multirow{4}{*}{87.1} & \multirow{4}{*}{-19.9} \\
\hline & & 30 & $5.9 \times 10^{-4}$ & & & \\
\hline & & 40 & $1.9 \times 10^{-3}$ & & & \\
\hline & & 50 & $5.0 \times 10^{-3}$ & & & \\
\hline \multirow{4}{*}{2.0} & \multirow{4}{*}{0.0} & 20 & $1.4 \times 10^{-4}$ & \multirow{4}{*}{63.6} & \multirow{4}{*}{61.1} & \multirow{4}{*}{-110.2} \\
\hline & & 30 & $3.3 \times 10^{-4}$ & & & \\
\hline & & 40 & $7.6 \times 10^{-4}$ & & & \\
\hline & & 50 & $1.5 \times 10^{-3}$ & & & \\
\hline \multirow{4}{*}{2.0} & \multirow{4}{*}{200} & 20 & $1.2 \times 10^{-4}$ & \multirow{4}{*}{67.8} & \multirow{4}{*}{65.7} & \\
\hline & & 30 & $3.3 \times 10^{-4}$ & & & -954 \\
\hline & & 40 & $8.0 \times 10^{-4}$ & & & 50.4 \\
\hline & & 50 & $1.6 \times 10^{-3}$ & & & \\
\hline & & 20 & $1.5 \times 10^{-4}$ & & & \\
\hline 20 & 600 & 30 & $3.0 \times 10^{-4}$ & 668 & 644 & 093 \\
\hline 2.0 & 000 & 40 & $8.2 \times 10^{-4}$ & 00.0 & 04.4 & -99.0 \\
\hline & & 50 & $1.8 \times 10^{-3}$ & & & \\
\hline & & 20 & $1.5 \times 10^{-4}$ & & & \\
\hline 20 & 1000 & 30 & $3.4 \times 10^{-4}$ & 646 & 614 & -1092 \\
\hline 2.0 & 1000 & 40 & $6.7 \times 10^{-4}$ & 04.0 & 01.4 & -109.2 \\
\hline & & 50 & $1.9 \times 10^{-3}$ & & & \\
\hline
\end{tabular}

tail structure that swings randomly with temperature. This continuously would lead to bond breakage of $\mathrm{Fe}-\mathrm{O}_{3} \mathrm{~S}-$ bond which might expose the next layer to more acid effect and more corrosion leading to higher rates of corrosion (Scheme 2). Thus, two mutual effects control the rate of corrosion, first the inhibition effect through increasing the concentration of inhibitor that may be adsorbed on the surface of metal forming protective layer and second, the effect of temperature increase that weakens adsorption process, aids in $\mathrm{Fe}-\mathrm{O}_{3} \mathrm{~S}$ - bond cleavage and leads to increase in rate of corrosion.

These two mutual and counter effects were very clear in the activation energy values where the difference in activation energies between $0.0 \mathrm{ppm}$ and $1000 \mathrm{ppm}$ inhibitor was very small. This small difference was attributed to the effect of temperature that enhances rate of corrosion on the expense of the inhibitor effect. Experimentally, at $50^{\circ} \mathrm{C}$ using $1000 \mathrm{ppm}$ inhibitor, larger amount of hydrogen gas were evolved with respect to that at $0.0 \mathrm{ppm}$ which confirms enhancement of corrosion rate as a result of temperature over the inhibitor action.

Apparent entropy of activation shows that at larger inhibitor concentration, smaller $\Delta S^{\neq}$was obtained in $1.0 \mathrm{M}$ $\mathrm{HCl}$, whereas at larger inhibitor concentration, larger $\Delta S^{\neq}$ was obtained in $2.0 \mathrm{M} \mathrm{HCl}$ solution. This could be attributed to decrease or increase in solvent entropy as a result of desorption of water molecules that were adsorbed on the surface of the metal which were followed by adsorption of inhibitor on the surface of the metal as follows [29]:

$$
\text { Inhib.(sol.) }+\mathrm{H}_{2} \mathrm{O}_{(\text {ads. })} \longrightarrow \text { Inhib.(ads.) }+\mathrm{H}_{2} \mathrm{O}_{(\text {(sol.) }} \text {. }
$$

The enthalpy of activation values was in accordance with activation energy values. All values were of endothermic nature due to iron dissolution. 


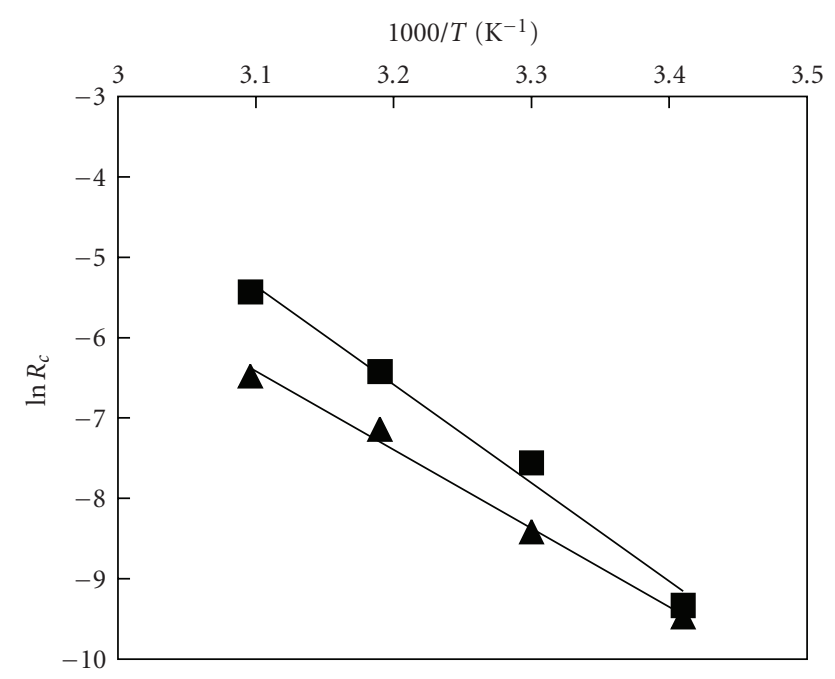

$2 \mathrm{M} \mathrm{HCl}$
$1 \mathrm{M} \mathrm{HCl}$

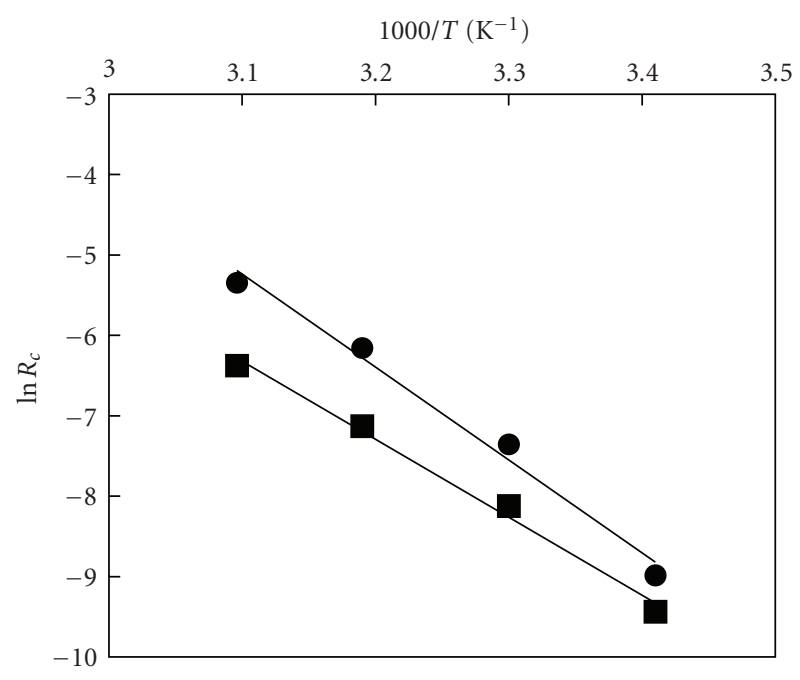

$2 \mathrm{M} \mathrm{HCl}$

- $1 \mathrm{M} \mathrm{HCl}$

(a)

(b)

FIgURE 3: Arrhenius plot of $\ln R_{c}$ versus reciprocal temperature $(1 / T)$ for (a) [LAS] $=0.0 \mathrm{ppm}$ and (b) [LAS] $=1000 \mathrm{ppm}$ at different $\mathrm{HCl}$ concentrations.

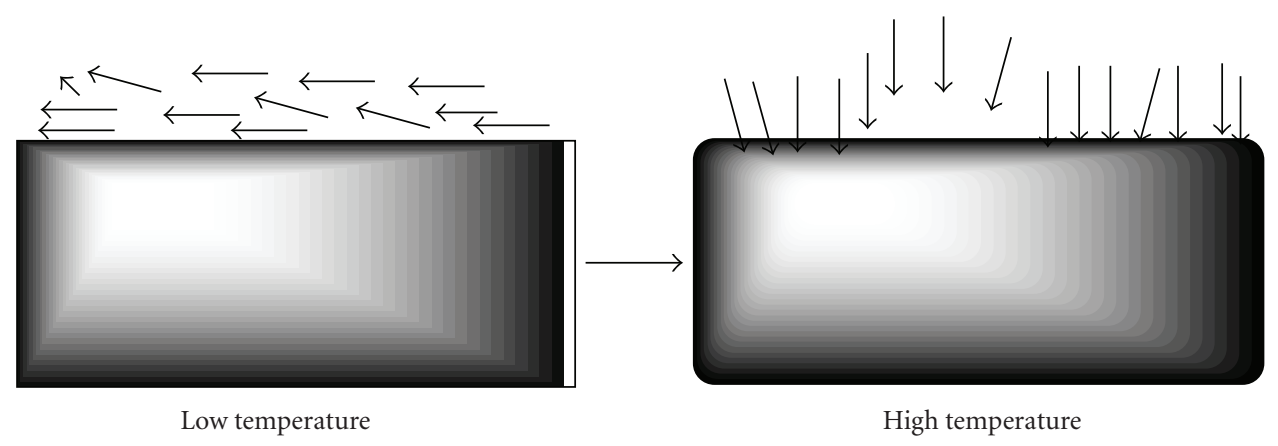

Scheme 2: Arrows show the orientation of inhibitor structure on the surface of iron.

3.2.2. In Presence of Magnetic Field. Table 2 shows the kinetic and thermodynamic parameters determined in the presence of magnetic field, respectively.

The rates of corrosion were relatively lower in presence of magnetic field, and consequently larger activation energies were determined. The corrosion rate lowering together with activation energy increase came in accordance with the shift to higher inhibition efficiency (i.e., \%IE) in presence of magnetic field (Table 2). Furthermore, by comparing solvent entropy change of activation (i.e., $\Delta S^{\neq}$) in absence and presence of magnetic field, it could be clearly seen that there exists a slight solvent entropy increase (i.e., surroundings) in presence of magnetic field. This necessarily imposes slightly larger entropy decrease in the system (Iron sheet with inhibitor), according to 2nd law of thermodynamics, which supports our saying that there had been more alignment, more orientation of inhibitor structure on the surface of the metal in presence of magnetic field that led to lower entropy values of the system (Iron sheet with inhibitor).
3.3. Adsorption Isotherms. Careful investigation performed for Tempkin [19, 20], Langmuir [21], and Frumkin [22] isotherms (8)-(10) would show the most fitting isotherm with maximum regression coefficients, $R^{2}$, using the following relationships:

$$
\begin{gathered}
\theta=\left(\frac{1}{f}\right) \ln \left(K_{\text {ads. }}[\text { LAS }]\right), \\
\log \left(\frac{\theta}{1-\theta}\right)=\log K_{\text {ads. }}+\log [\text { LAS }], \\
\log \left(\frac{\theta}{(1-\theta)[\text { LAS }]}\right)=\log K_{\text {ads. }}+g \theta,
\end{gathered}
$$

where $\theta$ is the degree of surface coverage of inhibitor on iron sheet surface, $K_{\text {ads. }}$ is equilibrium constant of the adsorption process, $g$ is adsorbate interaction parameter, and $f$ is heterogeneous factor of metal surface. The most fitting 


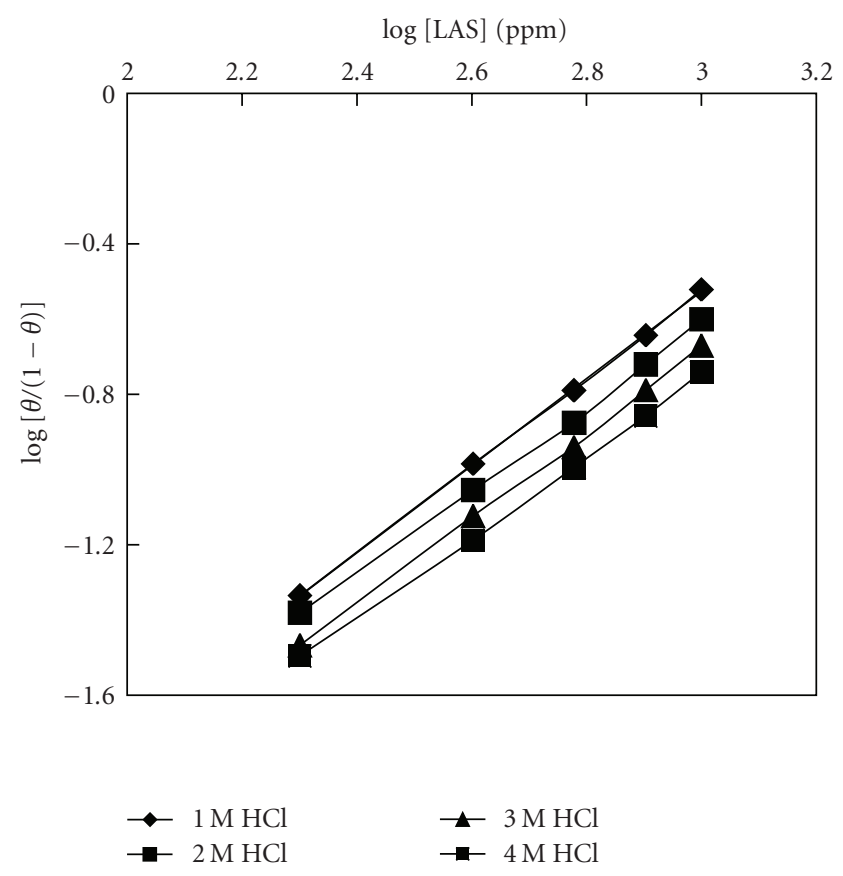

(a)

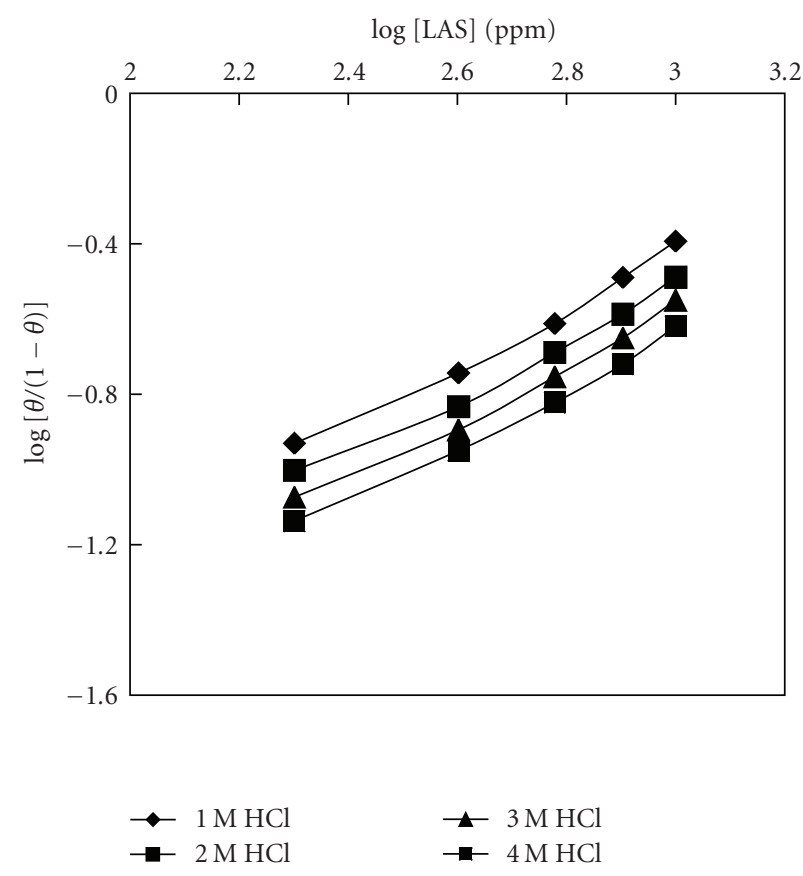

(b)

Figure 4: Langmuir isotherm curves (a) in the absence of magnetic field and (b) in the presence of magnetic field.

TABLE 2: Kinetic and thermodynamic parameters of corrosion in $\mathrm{HCl}$ solution in the presence of magnetic field.

\begin{tabular}{|c|c|c|c|c|c|c|}
\hline$[\mathrm{HCl}](\mathrm{M})$ & [LAS] $(\mathrm{ppm})$ & $\mathrm{T}\left({ }^{\circ} \mathrm{C}\right)$ & $R_{C}\left(\mathrm{mg} / \mathrm{cm}^{2} \cdot \mathrm{sec}.\right)$ & $E_{a}(\mathrm{~kJ} / \mathrm{mol})$ & $\Delta H^{\neq *}(\mathrm{~kJ} / \mathrm{mol})$ & $\Delta S^{\neq}(\mathrm{J} / \mathrm{mol} \cdot \mathrm{K})$ \\
\hline 1.0 & 0.0 & 20 & $1.2 \times 10^{-4}$ & 92.5 & 90.1 & -12.5 \\
\hline 1.0 & 200 & 20 & $1.5 \times 10^{-4}$ & 86.3 & 83.9 & -31.8 \\
\hline 1.0 & 600 & 20 & $2.3 \times 10^{-4}$ & 78.1 & 75.7 & -56.2 \\
\hline 1.0 & 1000 & 20 & $1.6 \times 10^{-4}$ & 90.3 & 87.9 & -17.6 \\
\hline 2.0 & 0.0 & 20 & $1.3 \times 10^{-4}$ & 64.1 & 61.7 & -108.7 \\
\hline 2.0 & 200 & 20 & $1.1 \times 10^{-4}$ & 68.0 & 65.6 & -96.8 \\
\hline 2.0 & 600 & 20 & $1.4 \times 10^{-4}$ & 67.0 & 64.6 & -98.2 \\
\hline 2.0 & 1000 & 20 & $1.4 \times 10^{-4}$ & 64.7 & 62.3 & -106.0 \\
\hline
\end{tabular}

*Calculated based on the assumption of unimolecular reaction where $E_{a}=\Delta H^{\neq}+\mathrm{RT}[25]$.

TABLE 3: Equilibrium constant and free energy of adsorption values for $1.0 \mathrm{hr}$ in presence and absence of magnetic field.

\begin{tabular}{lcccc}
\hline$[\mathrm{HCl}](\mathrm{M})$ & $\mathrm{T}\left({ }^{\circ} \mathrm{C}\right)$ & $K_{\text {ads. }}\left(\mathrm{M}^{-1}\right)$ & $\Delta G_{\text {ads. }}(\mathrm{kJ} / \mathrm{mol})$ & Magnetic field \\
\hline 1.0 & 20 & 125.0 & -21.6 & Present \\
1.0 & 20 & 109.6 & -21.2 & Absent \\
1.0 & 30 & 0.287 & -7.0 & Absent \\
1.0 & 40 & $2.5 \times 10^{-5}$ & 17.1 & Absent \\
1.0 & 50 & $1.1 \times 10^{-5}$ & 19.9 & Absent \\
\hline
\end{tabular}

curve was Langmuir isotherms with 0.988-0.999 regression coefficients as depicted in Figure 4.

Equilibrium constant $\left(K_{\text {ads. }}\right)$ of adsorption process determined using (9) could be further used to determine free energy of adsorption as follows:

$$
\Delta G_{\text {ads. }}=-\mathrm{RT} \ln \left(55.5 K_{\text {ads. }}\right),
$$

where 55.5 is the molar concentration of water, $R$ is the universal gas constant, and $T$ is the thermodynamic temperature. Table 3 summarizes the equilibrium constant and free energy of adsorption values in presence and absence of magnetic field.

The spontaneity of the adsorption process was decreased upon temperature increase, it even became nonspontaneous at 40 and $50^{\circ} \mathrm{C}$. The elevated temperature had an adverse effect on adsorption process, where inter- and intramolecular forces such as, electrostatic bond, coordinative bond, and even weaker interactions such as, $\pi-\pi$ stacking interactions were weaken, loosened, and eventually decomposed (Scheme 2). The sudden and enormous decrease of equilibrium constant at $30^{\circ} \mathrm{C}$ and higher temperatures confirms that adsorption is not favorable due to larger thermal energy on iron sheet, which prohibits adsorption process simultaneously. On the other hand, presence of magnetic field leads to larger equilibrium constant value (i.e., larger 
concentration of inhibitor adsorbed) and lower free energy value (i.e., more spontaneity). This could be explained on the basis of capability of magnetic field to align adsorbed and accumulated molecules on the surface of metal, which decreases free volume of metal exposed to $\mathrm{HCl}$ solution, and eventually resist corrosion and form better shielding layers.

\section{Conclusions}

The use of linear alkyl benzene sulphonate, LAS, as corrosion inhibitor on the surface of iron metal in the absence and presence of magnetic field and in acidic media was investigated. The most fitting isotherms in the adsorption of inhibitor molecules at surface of iron were Langmuir isotherms in acidic media. In the absence of magnetic field, the inhibitory effect of the LAS on Iron was clarified using the apparent activation energy values $\left(E_{a}\right)$; similarly, apparent entropy of activation showed that at larger inhibitor concentration solvent entropy decreases in $1.0 \mathrm{M} \mathrm{HCl}$ and solvent entropy increases in $2.0 \mathrm{M} \mathrm{HCl}$. At elevated temperatures, sudden spontaneity declines down, detected through equilibrium constant $\left(K_{\text {ads. }}\right)$ values and free energy value $\left(\Delta G_{\text {ads. }}\right)$ due to weakening and decomposition of inter- and intramolecular interactions. In the presence of magnetic field, lower rates of corrosion, and larger activation energies, solvent entropy increases and consequent larger entropy decreases in the system (Iron sheet with inhibitor). Furthermore, consequent larger spontaneity, through larger equilibrium constant $\left(K_{\text {ads. }}\right)$ value and lower free energy value, $\left(\Delta G_{\text {ads. }}\right)$ were detected.

\section{Acknowledgment}

Jordan University of Science \& Technology and Al-Balqa Applied University are acknowledged for this research work.

\section{References}

[1] H. Ju, Z.-P. Kai, and Y. Li, "Aminic nitrogen-bearing polydentate Schiff base compounds as corrosion inhibitors for iron in acidic media: a quantum chemical calculation," Corrosion Science, vol. 50, no. 3, pp. 865-871, 2008.

[2] G. Schmitt, "Application of inhibitors for acid media," British Corrosion Journal, vol. 19, no. 4, pp. 165-176, 1984.

[3] P. J. Sykes, "25 years of progress in electrochemical methods," British Corrosion Journal, vol. 25, pp. 175-183, 1993.

[4] S. Kertit, J. Aride, A. Ben-Bachir, A. Sghiri, A. Elkholy, and M. Etman, "Chemical and electrochemical inhibition studies of corrosion and hydrogen surface embrittlement. I. $\mathrm{Fe} 0.78 \mathrm{~B} 0.13 \mathrm{Si0} 09$ amorphous alloy in molar HCl," Journal of Applied Electrochemistry, vol. 19, no. 1, pp. 83-89, 1989.

[5] S. S. Abd El-Rehim, M. A. M. Ibrahim, and K. F. Khaled, "4aminoantipyrine as an inhibitor of mild steel corrosion in $\mathrm{HCl}$ solution," Journal of Applied Electrochemistry, vol. 29, no. 5, pp. 593-599, 1999.

[6] P. R. Roberge, "Corrosion inhibitors," in Handbook of Corrosion Engineering, pp. 833-862, McGraw-Hill, New York, NY, USA, 1999.

[7] J. O. M. Bockris and A. K. N. Reddy, Modern Electrochemistry, vol. 2, Plenum Publishing, New York, NY, USA, 1976.
[8] S. Muralidharan, M. A. Quraishi, and S. V. K. Iyer, "The effect of molecular structure on hydrogen permeation and the corrosion inhibition of mild steel in acidic solutions," Corrosion Science, vol. 37, no. 11, pp. 1739-1750, 1995.

[9] S. K. Shukla and M. A. Quraishi, "Cefotaxime sodium: a new and efficient corrosion inhibitor for mild steel in hydrochloric acid solution," Corrosion Science, vol. 51, no. 5, pp. 1007-1011, 2009.

[10] L. A. Hernandez-Alvarado, L. S. Hernandez, J. M. Miranda, and O. Dominguez, "The protection of galvanised steel using a chromate-free organic inhibitor," Anti-Corrosion Methods and Materials, vol. 56, no. 2, pp. 114-120, 2009.

[11] T. Hryniewicz, K. Rokosz, and R. Rokicki, "Electrochemical and XPS studies of AISI 316L stainless steel after electropolishing in a magnetic field," Corrosion Science, vol. 50, no. 9, pp. 2676-2681, 2008.

[12] R. A. Tacken and L. J. J. Janssen, "Applications of magnetoelectrolysis," Journal of Applied Electrochemistry, vol. 25, no. 1, pp. $1-5,1995$.

[13] V. C. Noninski, "Magnetic field effect on copper electrodeposition in the Tafel potential region," Electrochimica Acta, vol. 42, no. 2, pp. 251-254, 1997.

[14] O. Aaboubi, J. P. Chopart, J. Douglade, A. Olivier, C. Gabrielli, and B. Tribollet, "Magnetic field effects on mass transport," Journal of the Electrochemical Society, vol. 137, no. 6, pp. 17961804, 1990.

[15] J. Lee, S. R. Ragsdale, X. Gao, and H. S. White, "Magnetic field control of the potential distribution and current at microdisk electrodes," Journal of Electroanalytical Chemistry, vol. 422, no. 1-2, pp. 169-177, 1997.

[16] K. Shinohara and R. Aogaki, "Magnetic field effect on copper corrosion in nitric acid," Electrochemistry, vol. 67, no. 2, pp. 126-131, 1999.

[17] A. Ručinskien, G. Bikulčius, L. Gudavičiute, and E. Juzeliunas, "Magnetic field effect on stainless steel corrosion in $\mathrm{FeCl}_{3}$ solution," Electrochemistry Communications, vol. 4, no. 1, pp. 86-91, 2002.

[18] E. Samper, M. Rodríguez, M. A. De la Rubia, and D. Prats, "Removal of metal ions at low concentration by micellarenhanced ultrafiltration (MEUF) using sodium dodecyl sulfate (SDS) and linear alkylbenzene sulfonate (LAS)," Separation and Purification Technology, vol. 65, no. 3, pp. 337-342, 2009.

[19] M. I. Tempkin, Zhurnal Fizicheskoi Khimii, vol. 15, p. 296, 1941.

[20] A. K. Maayta and N. A. F. Al-Rawashdeh, "Inhibition of acidic corrosion of pure aluminum by some organic compounds," Corrosion Science, vol. 46, no. 5, pp. 1129-1140, 2004.

[21] I. Langmuir, "The constitution and fundamental properties of solids and liquids. Part I. Solids," The Journal of the American Chemical Society, vol. 38, no. 2, pp. 2221-2295, 1916.

[22] A. N. Frumkin, "Electrocapillary curve of higher aliphatic acids and the state equation of the surface layer," Zeitschrift für Physikalische Chemie, vol. 116, p. 466, 1925.

[23] C. M. A. Brett, I. A. R. Gomes, and J. P. S. Martins, "The electrochemical behaviour and corrosion of aluminium in chloride media. The effect of inhibitor anions," Corrosion Science, vol. 36, no. 6, pp. 915-923, 1994.

[24] M. Bouklah, B. Hammouti, M. Lagrenée, and F. Bentiss, "Thermodynamic properties of 2,5-bis(4-methoxyphenyl)1,3,4-oxadiazole as a corrosion inhibitor for mild steel in normal sulfuric acid medium," Corrosion Science, vol. 48, no. 9, pp. 2831-2842, 2006. 
[25] I. Ahamad and M. A. Quraishi, "Bis (benzimidazol-2-yl) disulphide: an efficient water soluble inhibitor for corrosion of mild steel in acid media," Corrosion Science, vol. 51, no. 9, pp. 2006-2013, 2009.

[26] K. J. Laidler and J. H. Meiser, Physical Chemistry, HoughtonMifflin, New York, NY, USA, 3rd edition, 1999.

[27] F. S. de Souza and A. Spinelli, "Caffeic acid as a green corrosion inhibitor for mild steel," Corrosion Science, vol. 51, no. 3, pp. 642-649, 2009.

[28] A. Popova, E. Sokolova, S. Raicheva, and M. Christov, "AC and DC study of the temperature effect on mild steel corrosion in acid media in the presence of benzimidazole derivatives," Corrosion Science, vol. 45, no. 1, pp. 33-58, 2003.

[29] E. Emranuzzaman, T. Kumar, S. Vishwanatham, and G. Udayabhanu, "Synergistic effects of formaldehyde and alcoholic extract of plant leaves for protection of N80 steel in 15\%HCl," Corrosion Engineering Science and Technology, vol. 39, no. 4, pp. 327-332, 2004. 

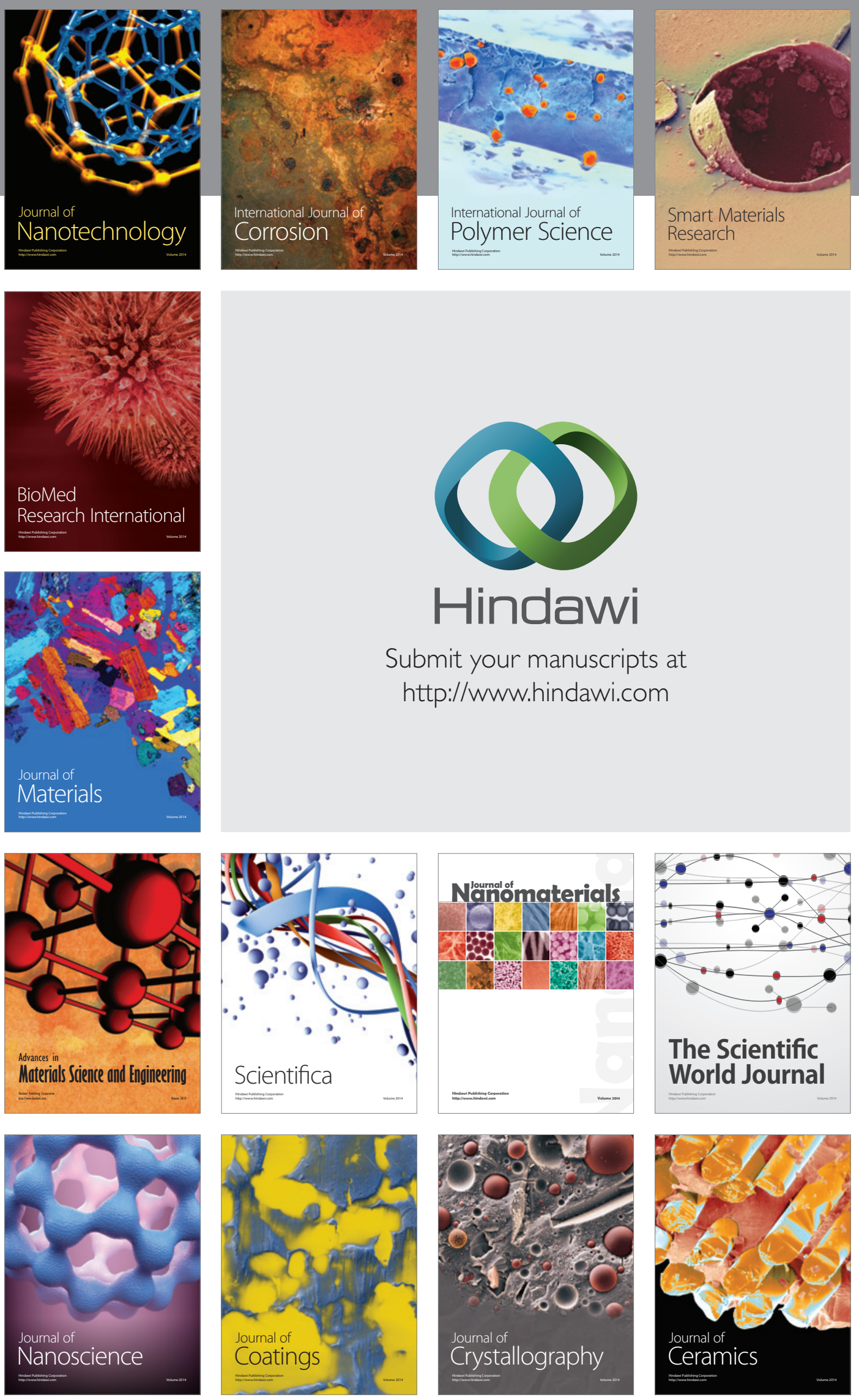

The Scientific World Journal

Submit your manuscripts at

http://www.hindawi.com

\section{World Journal}

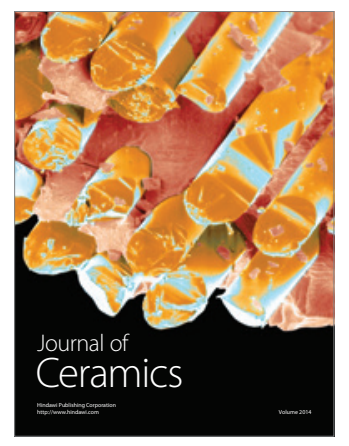

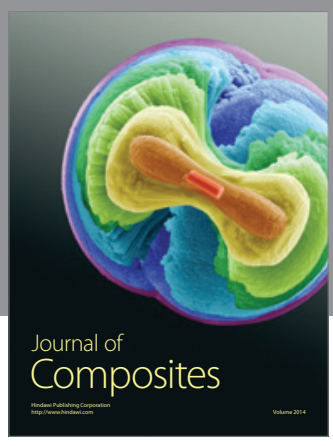
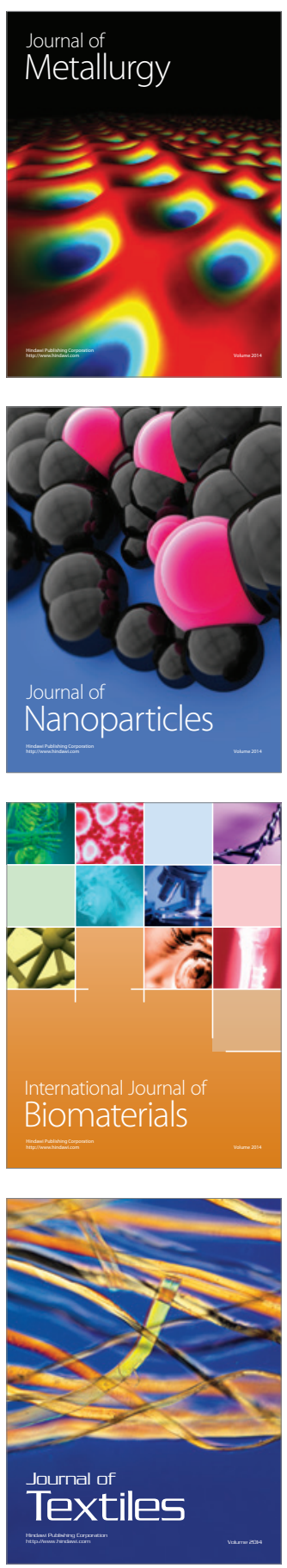